\title{
Scale-up Activation of Carbon Fibres for Hydrogen Storage
}

\author{
M. Kunowsky, J.P. Marco-Lozar, D. Cazorla-Amorós, A. Linares-Solano* \\ Grupo de Materiales Carbonosos y Medio Ambiente, Departamento de Química Inorgánica, Universidad de Alicante, Ap. 99, E-03080 Alicante, Spain
}

\begin{abstract}
In a previous study, we investigated, at a laboratory scale, the chemical activation of two different carbon fibres (CF), their porosity characterization, and their optimization for hydrogen storage [1]. In the present work, this study is extended to: (i) a larger range of $\mathrm{KOH}$ activated carbon fibres, (ii) a larger range of hydrogen adsorption measurements at different temperatures and pressures (i.e. at room temperature, up to $20 \mathrm{MPa}$, and at $77 \mathrm{~K}$, up to $4 \mathrm{MPa}$ ), and (iii) a scaling-up activation approach in which the obtained activated carbon fibres (ACF) are compared with those from laboratory-scale activation. The prepared samples cover a large range of porosities, which is found to govern their ability for hydrogen adsorption. The hydrogen uptake capacities of all the prepared samples have been analysed both in volumetric and in gravimetric bases. Thus, maximum adsorption capacities of around $5 \mathrm{wt} . \%$ are obtained at $77 \mathrm{~K}$, and $1.1 \mathrm{wt} . \%$ at room temperature, respectively. The packing densities of the materials have been measured, turning out to play an important role in order to estimate the total storage capacity of a tank volume. Maximum values of $17.4 \mathrm{~g} \mathrm{l}^{-1}$ at $298 \mathrm{~K}$, and $38.6 \mathrm{~g}^{-1}$ at $77 \mathrm{~K}$ were obtained.
\end{abstract}

Key words: activated carbon fibers, microporous materials, up-scaling, high pressure adsorption, gas storage, physisorption

\section{Introduction}

Hydrogen is considered an important vector for the future energy market. In this context, the storage of hydrogen plays a key role for mobile applications such as cars [2]. In the scope of the FreedomCAR initiative, the U.S. Department of Energy (DOE) specified a number of technological requirements which need to be achieved for a successful transition to hydrogen-based transportation [3]. The established targets set limits to e.g. the refilling time, the cycle life-time, the costs, and the densities of the storage system [3]. Ever since, these guidelines actuated as an important stimulation for research in this field. However, despite the worldwide effort of scientists and engineers, none of the current technologies meets all of these demands. Usually, in the research of materials for hydrogen storage, special attention is drawn to the gravimetric and volumetric densities. Especially in the case of introducing hydrogen technology into existing car designs, volumetric restrictions to the tank need to be taken into account [4]. Lately in 2009, the DOE revised their system density targets which are now based on light-duty vehicles and reduced them to $4.5 \mathrm{wt} . \%$ and $28 \mathrm{~g}_{\mathrm{H}_{2}} \mathrm{I}^{-1}$ (target for 2010), $5.5 \mathrm{wt} . \%$ and $40 \mathrm{~g}_{\mathrm{H}_{2}} \mathrm{l}^{-1}$ (target for 2015), and $7.5 \mathrm{wt} . \%$ and $70 \mathrm{~g}_{\mathrm{H}_{2}} \mathrm{1}^{-1}$ (ultimate target), respectively [5].

Presently, a number of methods and technologies are investigated regarding their hydrogen storage performance. Most of the current hydrogen car prototypes are based on high pressure vessels. This is a relatively simple and cost-efficient technology. However, the tanks occupy a lot of space due to low storage densities, and security concerns exist because of

\footnotetext{
${ }^{*}$ Corresponding author. Tel.: +34965903545; Fax: +34965903454

Email address: linares@ua.es (A. Linares-Solano)
}

the high pressures. For the compression, around $12 \%$ of the stored energy is consumed in order to reach $35 \mathrm{MPa}$, and $15 \%$ for $70 \mathrm{MPa}$, respectively [4]. Another possibility, which is favoured by some car manufacturers, is to store hydrogen as a liquid at cryogenic temperatures [6]. Although liquid hydrogen has a high density, the density of the storage system is reduced by the tank isolation and auxiliary devices. Even so, the isolation remains imperfect, causing some of the stored hydrogen to constantly evaporate. This fraction needs to be removed from the tank in order to avoid increasing pressures which would destroy the tank. In addition, a significant amount of its chemical energy (approximately one third) is needed in order to liquefy the hydrogen [3, 7]. A third method for increasing the low density of hydrogen is to bind it to materials. Because of the wide range of possible materials and reaction mechanisms, their categorization is sometimes debatable. However, one can distinguish between chemical and physical methods. In chemical methods, the $\mathrm{H}_{2}$ molecule is broken, and the elementary $\mathrm{H}$ atoms form chemical bonds with other atoms. Usually, for achieving reasonable gravimetric storage densities, high binding enthalpies are involved. These lead to a considerable amount of heat that needs to be transferred during charging and discharging [4, 8, 9]. Thus, for a typical heat of formation of $25 \mathrm{MJ} \mathrm{kg}_{\mathrm{H}_{2}}^{-1}$, a heat exchanger of several hundreds of $\mathrm{kW}$ would be necessary [4]. This significantly reduces the energy efficiency of the whole process, e.g. in the case of $\mathrm{MgH}_{2}$ by a 30\% [10]. In physical methods, in contrast, the $\mathrm{H}_{2}$ molecules are attracted to the surface of highly porous materials by Van-der-Waals interactions. Here, relatively low adsorption enthalpies of around 4 to $8 \mathrm{~kJ} \mathrm{~mol}^{-1}$ are involved, avoiding the need for active cooling devices [11, 12]. However, additionally 
to filling a tank with the adsorbent, high pressures and/or low temperatures are necessary in order to favour this physisorption process. A promising candidate among the different adsorbent materials are activated carbons. Through activation, highly porous materials with large specific surface areas can be prepared [13-17]. Due to their high porosity, activated carbon materials are able to adsorb considerable amounts of hydrogen $[1,7,12,18-34]$.

Carbon is the basis of organic compounds and is therefore an abundant element on earth which can be obtained from numerous sources. Thus, some of the advantages of carbon-based hydrogen storage materials are their high availability and low costs [18, 35]. After all, millions of tons of hydrogen storage material will be necessary in order to replace the number of existing cars with others based on hydrogen technology [4, 36]. In this sense, it is important to investigate the possibility of producing activated carbon materials on a larger scale [4, 32]. However, it is a difficult task to produce highly specialized adsorbents in large amounts. Thereby, the heat treatment is the most crucial step which requires careful control [16]. In addition, information about scale-up synthesis is poorly described in the open literature, due to the confidential character of commercial production data [37]. Recently, El Qada et al. compared physical activations of bituminous coal in a tube furnace and in a rotary kiln [38]. For hydrogen purification, Lopes et al. compared physical activation of activated carbons prepared at laboratory-scale with those obtained from a scale-up process [39]. The porosities in those approaches were reproducible within a margin of less than $6 \%$ [38], and around $9 \%$ [39], respectively.

The objective of the present work is twofold:

On the one hand, a scale-up approach is performed in order to produce optimized activated carbon fibre materials for hydrogen storage. Thereby, results obtained in a previous study are taken into account. In this preliminary work, we investigated the chemical activation on laboratory-scale of two different carbon fibres which were carbonized at different temperatures (973 K and $1273 \mathrm{~K}$, respectively), as well as their porosity optimization for hydrogen storage [1]. In the present study, the same two carbon fibre precursors are used for scale-up activation. The results from those activations are evaluated, and compared with those obtained from laboratory-scale activation. In our previous study, both, $\mathrm{KOH}$ and $\mathrm{NaOH}$, were used as activating agents [1]. The present study is restricted to activations with $\mathrm{KOH}$, because the activation process with $\mathrm{KOH}$ showed a higher yield in comparison with $\mathrm{NaOH}$ activation and featured a more appropriate, narrower porosity [1].

Secondly, extensive hydrogen adsorption measurements are carried out at both, room temperature (up to $20 \mathrm{MPa}$ ) and $77 \mathrm{~K}$ (up to $4 \mathrm{MPa}$ ), respectively. The adsorption amounts, obtained in a wide range of samples, are correlated with their narrow and total microporosities. Furthermore, the packing densities of all the samples prepared are measured, and the total hydrogen storage, which is the most important parameter from an application point of view, is assessed. As a result, the total hydrogen storage includes, apart from the adsorbed phase, the hydrogen that is compressed inside of the void space. Thereby, the impor- tance of the packing density of the material and of its measure is pointed out.

Finally, it is important to note that, in difference to other studies [7, 12, 18, 23-34], all the samples of the present study come from a given precursor. Thus, the possible effects of other pristine sample properties like for example the materials macrostructure, ash-content, etc. are eliminated.

\section{Materials and methods}

\subsection{Materials}

The two carbon fibre (CF) precursors, produced from coaltar pitch, were provided by Osaka Gas Co., Ltd (Osaka, Japan) and carbonized at different temperatures: Donacarbo S-241 at $1273 \mathrm{~K}$, and Donacarbo SL-242 at $973 \mathrm{~K}$, respectively. In order to activate them, the CFs were physically mixed with corresponding amounts of activating agent. For all activations, potassium hydroxide pellets (PRS Panreac) were used as activating agent. After the activation process, the activated carbon fibres $(\mathrm{ACF})$ were repeatedly washed and vacuum filtered, in order to remove the by-products of the reaction. First, $5 \mathrm{M}$ solution of hydrochloric acid, and then distilled water were used for washing. Finally, the washed ACFs were dried at $383 \mathrm{~K}$ overnight.

Concerning the nomenclature of the investigated materials, the first part of the sample names indicates the precursor which was used and corresponds to its carbonization temperature in centigrades. Thus, "D1000" corresponds to the samples that are based on the fibres which were carbonized at $1000^{\circ} \mathrm{C}$ (Donacarbo S-241), and "D700" to those which were carbonized at $700^{\circ} \mathrm{C}$ (Donacarbo SL-242), respectively. For denomination of the ACFs, a suffix like "-KX" is used, whereby the term " $X$ " indicates the $\mathrm{KOH}$-to- $\mathrm{CF}$ ratio (weight basis) that was used for activation. For example, for synthesising the sample D700-K4, the D700 precursor was activated with a $\mathrm{KOH}-$ to-CF ratio of $4: 1$. In addition, samples that were synthesized by scale-up activation are indicated by the term "-SU". Thus, the D1000-K6-SU sample is based on the D1000 CFs which were activated in a scale-up approach using a $\mathrm{KOH}-$ to- $\mathrm{CF}$ ratio of $6: 1$.

\subsection{Lab-scale activation}

For activations on laboratory scale ("lab-scale"), physical mixtures of $2 \mathrm{~g}$ of $\mathrm{CFs}$ with corresponding amounts of $\mathrm{KOH}$ pellets were prepared. Thereby, $\mathrm{KOH}$-to- $\mathrm{CF}$ ratios (weight basis) of $1: 1,2: 1,3: 1,4: 1,6: 1$, and 10:1 were used for activations of the D1000 precursor, and 1:1, 2:1, 3:1, 4:1, and 5:1 for activations of D700. The mixtures were placed inside a steel tray which was then introduced into a horizontal tube furnace. A schematic diagram of the experimental setup can be found in Fig. 1. The quartz tube of the furnace had a length of $1.5 \mathrm{~m}$ and an inner diameter of $64 \mathrm{~mm}$. The furnace was heated with a rate of $5 \mathrm{~K} \mathrm{~min}^{-1}$ up to a maximum temperature of $1023 \mathrm{~K}$. This temperature was kept constant for 75 min. Afterwards, the furnace was cooled down by convection. During the complete thermal treatment the mixture was exposed to a nitrogen gas flow of $500 \mathrm{ml} \mathrm{min}{ }^{-1}$ which passed through the furnace tube from one end to the other. 


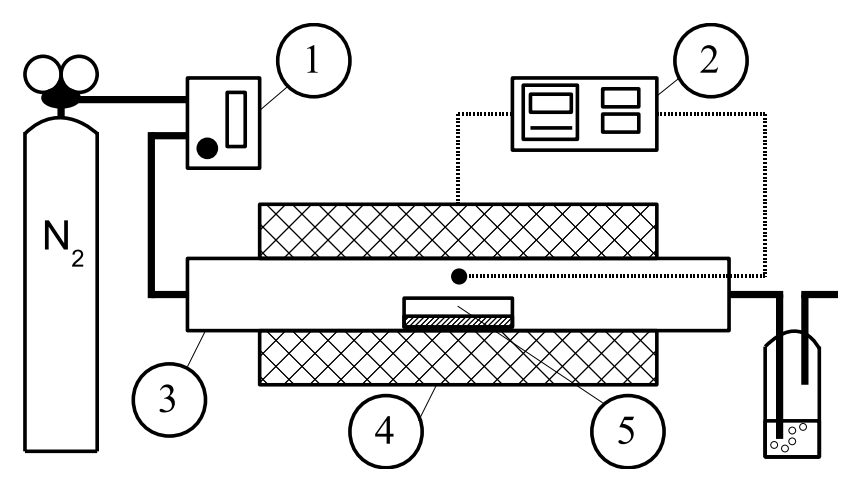

Figure 1: Schematic diagram of the tube furnace, used for lab-scale activations. (1) Flow controller, (2) temperature controller, (3) quartz tube, (4) tube furnace, (5) tray with sample.

\subsection{Scale-up activation}

In the case of scale-up activations, the amount of CF precursor was increased to $30 \mathrm{~g}$. Based on the lab-scale results (see below in Section 3) a KOH-to-CF ratio of 3:1 was used for activation of D700, and 6:1 for the D1000 precursor, respectively. For activations, a chamber furnace was used (see schematic diagram in Fig. 2). The inner dimensions of the chamber were: $250 \mathrm{~mm}$ (width), $350 \mathrm{~mm}$ (depth), $240 \mathrm{~mm}$ (height). Similar activating conditions as in the case of lab-scale activations were used for the thermal treatments. For each scale-up activation, the mixture of $\mathrm{CFs}$ and $\mathrm{KOH}$ pellets was equally portioned and placed into two ceramic trays. These were symmetrically placed into the center of the chamber. Due to the design of the furnace, different to the tube furnace used for the lab-scale activations, other conditions were used for the nitrogen gas flow. Thus, the flow rate was $3000 \mathrm{ml} \mathrm{min}{ }^{-1}$, and the flow passed from several outlet openings at the bottom to a single outlet at the top of the furnace chamber.

\subsection{Porosity characterization}

The CF precursors and ACF samples were characterized by nitrogen adsorption at $77 \mathrm{~K}$ and by carbon dioxide adsorption at $273 \mathrm{~K}$, respectively. The measurements were carried out using Quantachrome Autosorb-6 devices. Prior to adsorption isotherms, the samples were outgassed at $523 \mathrm{~K}$ for 4 hours under vacuum. From $\mathrm{N}_{2}$ adsorption isotherms, the apparent $\mathrm{BET}$ surface areas, $\mathrm{S}_{B E T}$, as well as the total micropore volumes, $\mathrm{V}_{D R}\left(\mathrm{~N}_{2}\right)$, were calculated. From $\mathrm{CO}_{2}$ adsorption isotherms, the volumes of narrow micropores smaller than $0.7 \mathrm{~nm}, \mathrm{~V}_{D R}\left(\mathrm{CO}_{2}\right)$, were obtained [40]. Both, total and narrow micropore volumes, were calculated using the DR (Dubinin-Radushkevich) equation.

\subsection{Packing densities}

The packing densities, $\delta_{\text {Pack }}$, of the samples were measured in a mechanical press. A known mass of sample was introduced into a cylindrical steel mould with a diameter of $13 \mathrm{~mm}$. In order to compact the sample, a force equivalent to the mass of 1 tonne was applied on the sample with a steel cylinder. The

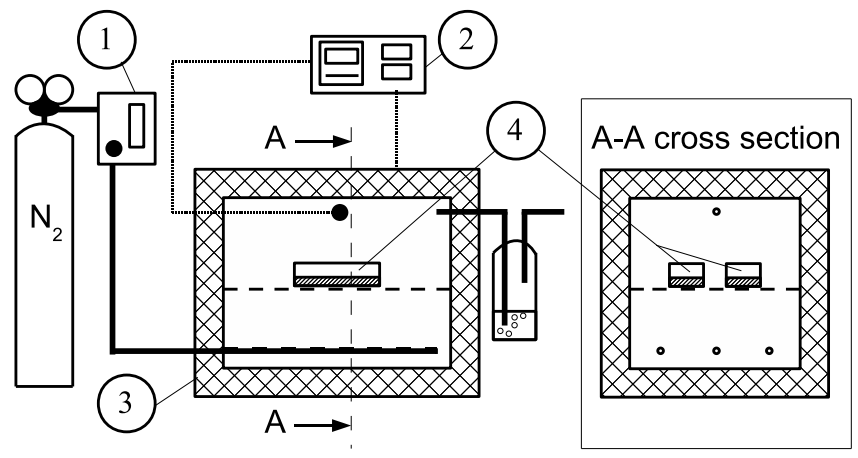

Figure 2: Schematic diagram of the chamber furnace, used for scale-up activations. (1) Flow controller, (2) temperature controller, (3) chamber furnace, (4) trays with sample.

volume occupied by the sample was evaluated by measuring the penetration depth of the steel cylinder in comparison with a reference measurement in which no sample was introduced into the mould.

\subsection{Hydrogen adsorption measurements}

Hydrogen adsorption was measured in two different devices. In the case of both experimental setups, hydrogen gas of 5.0 purity $(99.999 \%)$ was used for the measurements.

Room temperature measurements were carried out at $298 \mathrm{~K}$ with pressures up to $20 \mathrm{MPa}$ using a fully automated volumetric apparatus, designed and built up at University of Alicante [41]. Sample masses ranged between 0.5 and $1.5 \mathrm{~g}$. Previous to each measurement, the sample was heated at $423 \mathrm{~K}$ for $4 \mathrm{~h}$ under vacuum, and the weight of the degassed sample was measured. After that, the sample was located in the sample volume, where it was degassed again at $423 \mathrm{~K}$ during another $4 \mathrm{~h}$ in vacuum, in order to prepare the sample for the measurement. The device was regularly tested for leaks, and the reference volume $\left(<19 \mathrm{~cm}^{3}\right)$ was determined with a calibrated volume. The volume on the sample side was determined with helium gas before each measurement and ranged between 19.1 and $25.2 \mathrm{~cm}^{3}$. The reference temperature was maintained at $308 \mathrm{~K}$, while the sample volume temperature of $298 \mathrm{~K}$ was controlled by a thermostat with cooling liquid.

For hydrogen isotherms at $77 \mathrm{~K}$ and up to $4 \mathrm{MPa}$ a highpressure device (provided by DMT GmbH \& Co. KG) was used which incorporates a Sartorius 4406 microbalance. The weight of the measured samples ranged from around 0.4 to $0.7 \mathrm{~g}$. The samples were degassed at $423 \mathrm{~K}$ for $4 \mathrm{~h}$ under vacuum. After cooling by convection, the sample container of the balance was cooled with liquid nitrogen. Throughout the experiments the level of the nitrogen bath was kept constant. Reference measurements without sample were performed, and the experimental results were corrected for buoyancy effects related to the displacement of gas by the sample, the sample holder, and the pan [12, 23]. 


\section{Results}

\subsection{Sample characterization: Porosity, yields and densities}

Table 1 compiles the porosity characterization of the two precursors and of all the activated samples investigated in the present work together with their packing densities and activation yields. For the ACF samples, the activation conditions can be deduced from their denomination, as it has been explained before in Section 2.1. In Fig. 3, different parameter values from sample characterization are plotted against the $\mathrm{KOH}$-to-CF ratios. In Fig. 3a the change in apparent BET surface area is presented, while in Fig. $3 \mathrm{~b}$ the development of the narrow microporosity (the one obtained from $\mathrm{CO}_{2}$ adsorption, corresponding to micropores $<0.7 \mathrm{~nm}$ ) is shown. Finally, in Fig. 3cthe change of the activation process yield is shown for increasing $\mathrm{KOH}$-toCF ratios.

\subsubsection{Characterization of lab-scale samples}

In this Section, the samples which were activated on lab-scale (see Section 2.2) are investigated. In Fig. 3a, it can be observed that, for both precursors, the apparent BET surface area of the $\mathrm{ACFs}$ increases with the $\mathrm{KOH}-$ to- $\mathrm{CF}$ ratio. However, for similar KOH-to-CF ratios, samples which are activated from D700 precursors reach higher BET surface areas than those that are based on the D1000 precursor. For D700-based ACFs, the highest BET surface area, $\mathrm{S}_{B E T}$, close to $3000 \mathrm{~m}^{2} \mathrm{~g}^{-1}$ is obtained with the highest ratio of 5:1. In the case of D1000-based ACFs, a maximum value close to $2000 \mathrm{~m}^{2} \mathrm{~g}^{-1}$ is reached with the highest $\mathrm{KOH}$-to-CF ratio studied (10:1). It is worth mentioning that the BET surface area of the D1000-based ACFs increases significantly only up to a KOH-to-CF ratio of 6:1. In Table 1 it can be seen that the total microporosity, $\mathrm{V}_{D R}\left(\mathrm{~N}_{2}\right)$, follows a similar tendency as the BET surface area, $\mathrm{S}_{B E T}$.

Also for the development of the narrow microporosity it can be observed that the samples based on the D700 precursor reach higher values than those that are based on D1000 (see Fig. 3b). In the case of $\mathrm{D} 700$ based samples, the $\mathrm{V}_{D R}\left(\mathrm{CO}_{2}\right)$ micropore volume strongly increases up to a ratio of $3: 1$, reaching $0.79 \mathrm{~cm}^{3} \mathrm{~g}^{-1}$. For higher ratios, no clear trend can be observed. For D1000 based samples, the narrow microporosity increases up to a value of $0.67 \mathrm{~cm}^{3} \mathrm{~g}^{-1}$ for a ratio of $4: 1$. When the ratio is further increased, no significant change can be observed up to a ratio to $6: 1$. However, the narrow microporosity clearly decreases for a very large ratio of 10:1.

Fig. 3c shows that the yield of the ACFs decreases with the $\mathrm{KOH}-$ to-CF ratio. Thereby, a linear tendency can be observed for both, D700 and D1000. With increasing ratio, the difference in the performance of the two precursors becomes more explicit. Thus, using similar ratios, slightly higher yield values are obtained for D1000 in comparison with the D700 precursor.

The packing densities of the samples, listed in Table 1 show that the two $\mathrm{CF}$ precursors have the highest densities, which drop upon chemical activation. Interestingly, the density of the resulting ACFs are quite high. Thus, in the case of D1000based ACFs, the values fluctuate between 0.71 and $0.83 \mathrm{~g} \mathrm{~cm}^{-3}$. The packing densities of ACFs derived from the D700 precursor
Table 1: Characterization results of the carbon fibre precursors and the activated carbon fibres

\begin{tabular}{lccccc}
\hline Sample & $\begin{array}{c}\mathrm{S}_{B E T} \\
\mathrm{~m}^{2} \mathrm{~g}^{-1}\end{array}$ & $\begin{array}{c}\mathrm{V}_{D R}\left(\mathrm{~N}_{2}\right) \\
\mathrm{cm}^{3} \mathrm{~g}^{-1}\end{array}$ & $\begin{array}{c}\mathrm{V}_{D R}\left(\mathrm{CO}_{2}\right) \\
\mathrm{cm}^{3} \mathrm{~g}^{-1}\end{array}$ & $\begin{array}{c}\delta_{\text {Pack }} \\
\mathrm{g} \mathrm{cm}^{-3}\end{array}$ & $\begin{array}{c}\text { Yield } \\
\%\end{array}$ \\
\hline D1000 & - & - & 0.08 & 1.03 & - \\
D1000-K1 & 683 & 0.31 & 0.32 & 0.82 & 83 \\
D1000-K2 & 966 & 0.46 & 0.48 & 0.73 & 79 \\
D1000-K3 & 1288 & 0.59 & 0.57 & 0.83 & 74 \\
D1000-K4 & 1456 & 0.67 & 0.67 & 0.71 & 72 \\
D1000-K6 & 1894 & 0.89 & 0.65 & 0.76 & 56 \\
D1000-K10 & 1937 & 0.84 & 0.53 & 0.71 & 34 \\
\hline D1000-K6-SU & 2259 & 0.97 & 0.71 & 0.62 & 48 \\
\hline D700 & 7 & 0.00 & 0.11 & 0.99 & - \\
D700-K1 & 999 & 0.46 & 0.47 & 0.74 & 83 \\
D700-K2 & 1491 & 0.71 & 0.65 & 0.74 & 80 \\
D700-K3 & 2004 & 0.94 & 0.79 & 0.67 & 74 \\
D700-K4 & 2472 & 1.13 & 0.71 & 0.58 & 65 \\
D700-K5 & 2888 & 1.23 & 0.80 & 0.56 & 55 \\
\hline D700-K3-SU & 2364 & 1.02 & 0.79 & 0.63 & 60 \\
\hline
\end{tabular}

drop continuously with increasing degree of activation, reaching values between 0.56 and $0.74 \mathrm{~g} \mathrm{~cm}^{-3}$.

\subsubsection{Characterization of scale-up samples}

The results obtained for lab-scale activations, shown in the above Section, were the basis on which the $\mathrm{KOH}$-to-fibre ratios for the scale-up approaches were selected. The main criteria were an adequate packing density and porosity, optimized for hydrogen storage application at both, room and cryogenic temperatures, as well as reasonable activation yields. For the D1000 precursor, a KOH-to-fibre ratio of 6:1 was chosen, because high values were reached for both, total and narrow micropore volumes. In addition, a very high packing density was measured, and a reasonably high process yield was achieved. For scale-up activation of the D700 CF, a ratio of 3:1 was selected. Although higher BET surface areas were achieved with higher ratios, the narrow micropore volume already reached a very high value. Also the fact that both, packing density and process yield, dropped significantly when more activating agent was used, militated in favour of the 3:1 ratio.

The results for scale-up activations of the two precursors, D700 and D1000, are represented in Table 1 and in Fig. 3 by filled symbols. One of the objectives of the present work is to compare $\mathrm{KOH}$ activation in two scales (lab-scale and scale-up). Before extracting conclusions from such comparison, a study of the reproducibility of both systems is needed. Hence, a systematic reproducibility study of the $\mathrm{KOH}$ activation process has been first carried out in the lab-scale, using an anthracite precursor; 4 runs were done at a fixed $\mathrm{KOH}$-to-carbon ratio of $4: 1$. The results show porosity values within a margin of $4 \%$. Similarly, experiments were repeated in the scale-up system (in that case for both $\mathrm{CF}$ precursors), the porosity values being reproducible within a margin of $6 \%$. At a first glance, the data in Table 1 and Fig. 3 show a reasonable agreement between the results for both scale-up processes. A closer look reveals that 


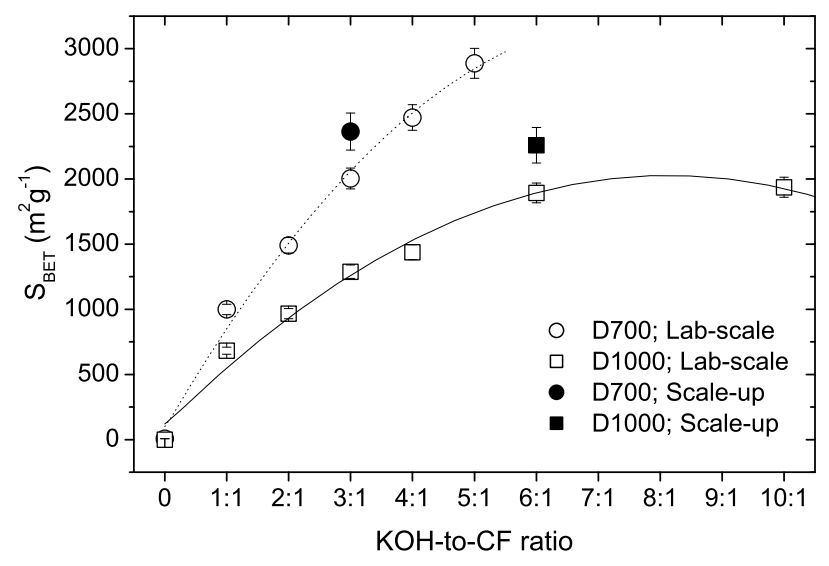

(a) BET surface area, $\mathrm{S}_{B E T}$

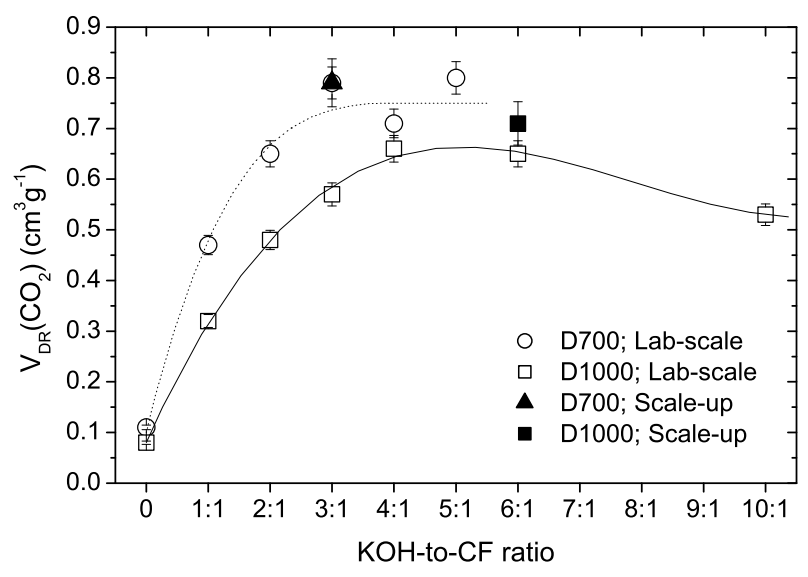

(b) Narrow microporosity, $\mathrm{V}_{D R}\left(\mathrm{CO}_{2}\right)$

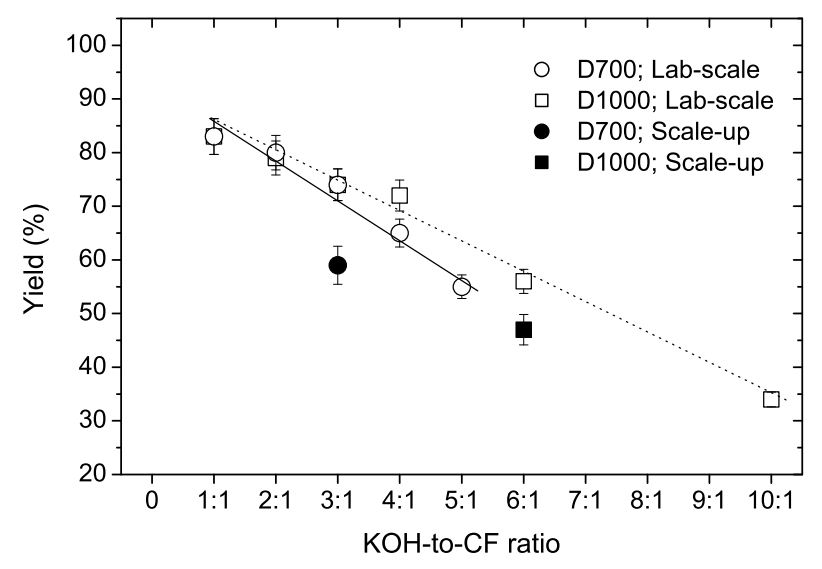

(c) Activation yield

Figure 3: Different characterization values plotted against KOH-to-fibre ratios for samples that were activated using D700 precursors (circles and triangle) and D1000 precursors (squares). Empty symbols indicate lab-scale activations, filled symbols indicate scale-up activations. The respective diagrams show (a) the BET surface area, $\mathrm{S}_{B E T}$, (b) the narrow microporosity, $\mathrm{V}_{D R}\left(\mathrm{CO}_{2}\right)$, and (c) the activation yield.

the BET surface areas of the synthesized materials reach around $2300 \mathrm{~m}^{2} \mathrm{~g}^{-1}$ (see Fig. 3a). These values are almost $10 \%$ higher, when compared to the lab-scale samples which were activated using similar $\mathrm{KOH}$-to- $\mathrm{CF}$ ratios. Also the narrow microporosity of the scale-up samples, shown in Fig. $3 \mathrm{~b}$, reaches higher values in comparison with those obtained from lab-scale activation. Thus, $0.79 \mathrm{~cm}^{3} \mathrm{~g}^{-1}$ are measured for the sample originating from the D700 precursor, and $0.71 \mathrm{~cm}^{3} \mathrm{~g}^{-1}$ for the activated D1000 sample. All the above commented differences should be ascribed to the two process scales, i.e. the different design of the activation systems, and not to reproducibility of the activation process itself whose influence is much less. Concerning the yields of the scale-up processes, which are included in Fig. 3c it is observed that: (i) the carbonization temperature does not significantly affect the yield values; and (ii) lower values are obtained for the scaled-up process in comparison with the corresponding lab-scale samples: $48 \%$ instead of $56 \%$ for the D1000 sample, and 60\% instead of 74\% for the D700 sample. Additionally, Table 1 shows that both scale-up samples have similar packing densities (over $0.6 \mathrm{~g} \mathrm{~cm}^{-3}$ ).

\subsection{Hydrogen adsorption results}

In comparison with other gases, hydrogen has a relatively low heat of adsorption of around 4-8 $\mathrm{kJ} \mathrm{mol}^{-1}$ [11, 12]. Therefore, hydrogen adsorption is favoured at high pressures and/or at low temperatures. Both of the scenarios were investigated in this study. In Section 3.2.1 hydrogen isotherms were measured at room temperature $(298 \mathrm{~K})$ and up to high pressures of $20 \mathrm{MPa}$; in Section 3.2.2, liquid nitrogen temperature $(77 \mathrm{~K})$ and pressures up to $4 \mathrm{MPa}$ were used.

\subsubsection{Hydrogen isotherms at room temperature}

The hydrogen adsorption isotherms of the investigated materials, measured at $298 \mathrm{~K}$, are shown in Fig. 4. In Fig. 4a the isotherms of the D1000 precursor, and of the ACF samples which were derived from it, are presented. The unactivated D1000 precursor already adsorbs over $0.3 \mathrm{wt} \%$. By chemical activation, the adsorption amount can be increased significantly. For the samples which were activated on lab-scale, the 


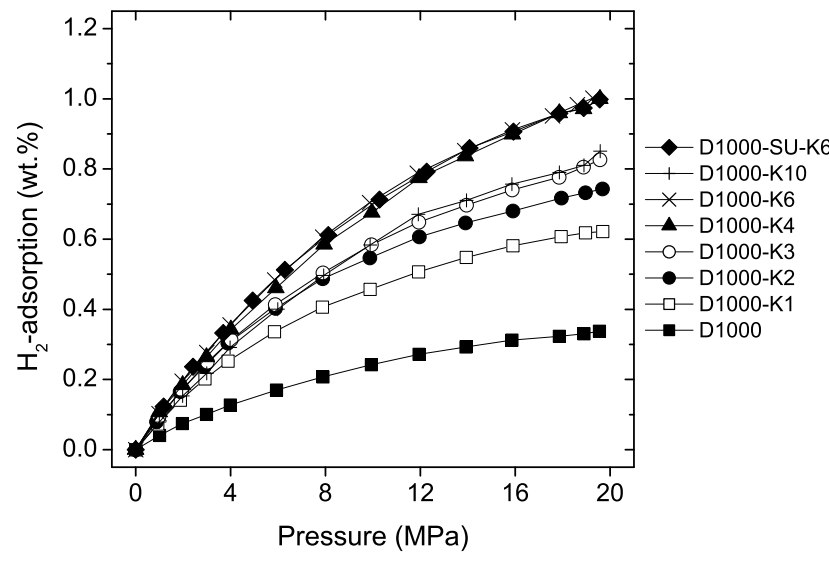

(a) D1000 samples

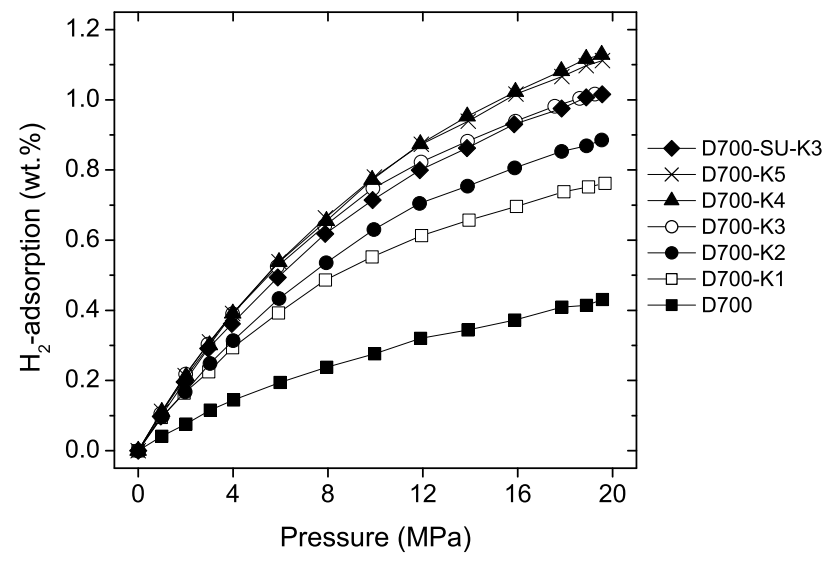

(b) D700 samples

Figure 4: $\mathrm{H}_{2}$ excess adsorption isotherms at room temperature for precursors, lab-scale samples, and scale-up (SU) samples based on (a) D1000 and (b) D700 fibres.

adsorption amount increases up to a KOH-to-CF ratio of 4:1, reaching $1 \mathrm{wt} . \%$. When the ratio is increased to $6: 1$, no significant increase can be observed. Remarkably, the adsorption amount decreases to around $0.8 \mathrm{wt} . \%$ when the ratio is further increased to 10:1. Probably, this is due to a destruction of the narrow microporosity which controls the hydrogen uptake at room temperature and $20 \mathrm{MPa}$ (see Section 4.2 below). The ACF that was obtained from scale-up activation performs very good in comparison with the lab-scale samples. Thus, a maximum adsorption amount of $1 \mathrm{wt} . \%$ is reached by this sample.

In Fig. 4b, isotherms of samples which are based on the D700 precursor are shown. The unactivated D700 CF performs better than the unactivated D1000, reaching over $0.4 \mathrm{wt} . \%$. Upon activation with $\mathrm{KOH}$, a strong increase of hydrogen adsorption can be observed. Interestingly, the activated D700 CFs perform better than those that were derived from D1000, as a consequence of the improved porosity development obtained with the D700 precursor. Similarly to what happened in the case of activated D1000, the adsorption amount increases with the KOH-to-CF ratio up to a ratio of $4: 1$. When the ratio is further increased to $5: 1$, no significant increase in hydrogen adsorption is observed. The maximum adsorption amount reached for lab-scale activated samples is $1.1 \mathrm{wt} . \%$. Also the scale-up sample that was derived from D700 shows good performance, reaching a maximum adsorption amount of $1 \mathrm{wt} . \%$.

\subsubsection{Hydrogen isotherms at $77 \mathrm{~K}$}

The hydrogen adsorption isotherms at $77 \mathrm{~K}$ of the samples based on D1000 and D700 are shown in Fig. 5 In Fig. 5a it can be observed that, for the unactivated D1000 CF, the values for hydrogen adsorption fluctuate around zero, and that its hydrogen adsorption isotherm shows adsorption equilibrium problems. This lack of adsorption equilibrium, caused by hydrogen diffusion problems, is in agreement with Table 1 where $\mathrm{N}_{2}$ adsorption could not be carried out. As expected, the adsorption increases significantly when the precursor is chemically activated with $\mathrm{KOH}$. Interestingly, the samples that were activated with $\mathrm{KOH}$-to-CF ratios of 3:1, 4:1, 6:1, and 10:1 reach similar adsorption amounts of around $3.4 \mathrm{wt} . \%$. On the other hand, the adsorption amount is significantly higher for the scale-up sample, reaching $4.5 \mathrm{wt} . \%$.

In contrast to what was observed for the D1000 precursor, the unactivated D700 CF already adsorbs $1 \mathrm{wt}$.\% of hydrogen at $77 \mathrm{~K}$ (see Fig. 5b). In difference to the former, D700 adsorbs a small amount of nitrogen at $77 \mathrm{~K}$ and has a small amount of narrow microporosity. By $\mathrm{KOH}$ activation, the adsorption amounts of the lab-scale samples continuously increase with the $\mathrm{KOH}$ to-CF ratio. The highest adsorption amount of around $5 \mathrm{wt} . \%$ is reached for a ratio of 5:1. Also the scale-up activated sample shows high hydrogen uptake, reaching more than $4.5 \mathrm{wt} . \%$.

\section{Discussion}

\subsection{Material synthesis}

The above presented results show that both CF precursors can be well activated with $\mathrm{KOH}$, leading to highly microporous adsorbents. The characteristics of the materials can be tuned by the amount of activating agent which is used. Also the carbonization temperature of the CF precursors has a high impact on the porosity development. Interestingly, it turned out that the $\mathrm{CF}$ which was carbonized at a lower temperature develops more porosity, even if less activating agent was used, and allows to develop better adsorbents having higher yields. These two observations are interesting from an economical point of view [1]. Apparently, the D1000 CF traversed a maximum porosity development concerning both, $\mathrm{S}_{B E T}$ and $\mathrm{V}_{D R}\left(\mathrm{CO}_{2}\right)$, for a $\mathrm{KOH}-$ to- $\mathrm{CF}$ ratio of around 6:1. The $\mathrm{D} 700$ precursor reaches very high $\mathrm{V}_{D R}\left(\mathrm{CO}_{2}\right)$ values already for smaller ratios. However, the narrow microporosity remains on a high level for larger ratios. On the other hand, the performance of the D700 precursor for developing high BET surface areas does not seem to be exhausted even for the highest $\mathrm{KOH}-\mathrm{to}-\mathrm{CF}$ ratio investigated in this study. All these results clearly show the advantage of the 


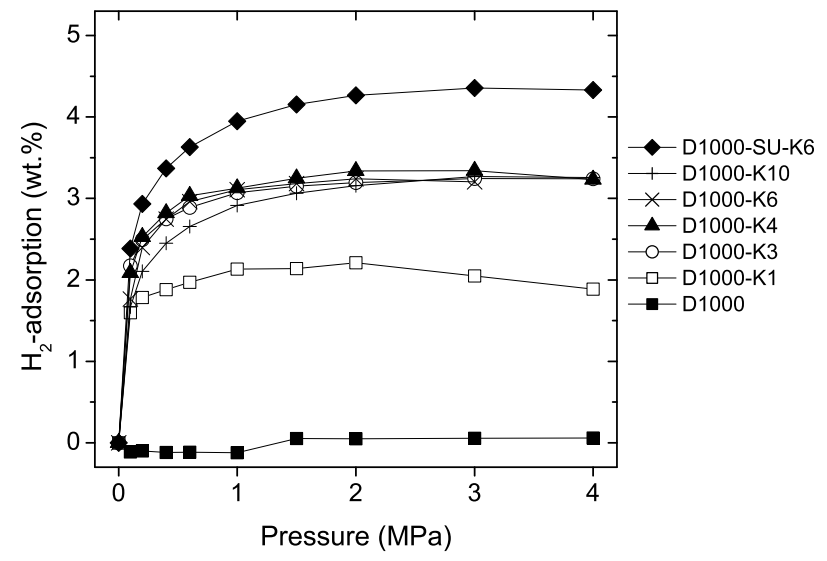

(a) D1000 samples

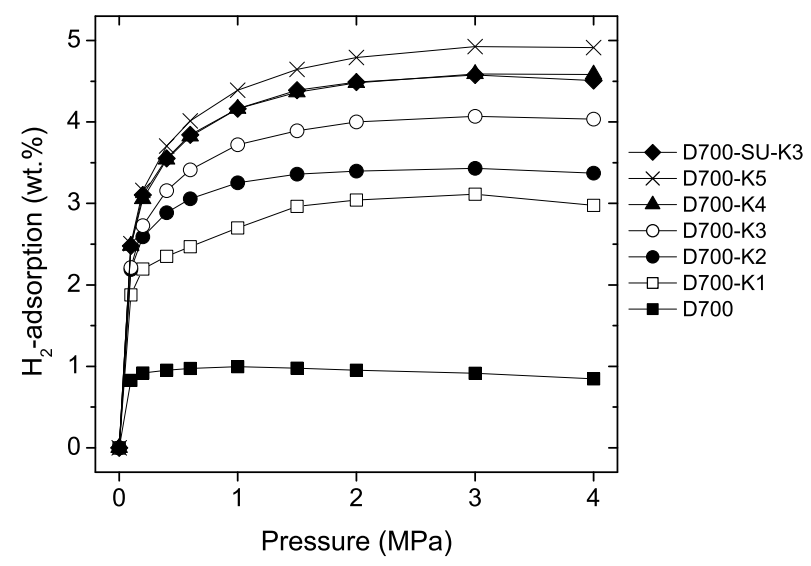

(b) D700 samples

Figure 5: $\mathrm{H}_{2}$ excess adsorption isotherms at $77 \mathrm{~K}$ for precursors, lab-scale samples, and scale-up (SU) samples based on (a) D1000 and (b) D700 fibres.

use of the lower carbonization temperature, and a much better performance of the D700 precursor in comparison with D1000.

Regarding the results from the scale-up activated samples, a general tendency can be observed. In comparison with labscale activations which use similar $\mathrm{KOH}-$ to- $\mathrm{CF}$ ratios, higher porosity is developed (see Fig. 3a and Fig. 3b), and lower process yields (Fig. 3c) are obtained. This indicates that a higher degree of activation took place during the scale-up approach. The different performance of lab-scale and scale-up activations can be explained by the specific conditions which predominate the activating processes in the two furnaces. For example, different nitrogen gas flows were used in the lab-scale and scaleup activations. The flow rate is known to have a high impact on the activation process [17, 42, 43]. It is responsible for eliminating gaseous reaction products which are formed upon chemical activation, thus enhancing the process [17, 42, 43]. However, we cannot solely assume a relation between the flow rate and the mass of the activated precursor. In fact, by doing so, we would obtain a higher specific flow rate for lab-scale activation $\left(250 \mathrm{ml}_{N_{2}}\left(\min \mathrm{g}_{C}\right)^{-1}\right)$ than for scale-up activation $\left(100 \mathrm{ml}_{N_{2}}\left(\min \mathrm{g}_{C}\right)^{-1}\right)$. Apparently, also the design of the furnaces has to be taken into account. In the case of the tube furnace, a laminar rather than a turbulent nitrogen gas flow is expected, due to the relatively small diameter of the tube in comparison with its length. Also the lower gas flow is advantageous for a laminar flow. On the other hand, a more turbulent gas flow is expected inside the chamber furnace, due to its design and the higher flow rate. Despite its lower controllability, a turbulent gas flow turned out to be advantageous for the activation process in the chamber furnace, possibly due to higher local flow velocities at the sample interfaces.

Future research on scale-up activation for production of ACs for hydrogen storage should focus on further scale-up of the activation process, for example in continuously operating furnaces of industrial scale.

\subsection{Hydrogen storage performance}

It is common practice to try to find correlations between porosity and hydrogen adsorption [12, 23-31]. In previous studies, it was found that at $298 \mathrm{~K}$ and $20 \mathrm{MPa}$ the gravimetric adsorption amount can be correlated with the narrow microporosity $(<0.7 \mathrm{~nm})[12,22-25]$. Fig. 6a confirms the linear relationship existing between the narrow micropore volume of the studied samples and their gravimetric hydrogen uptakes at room temperature. In the case of hydrogen adsorption at $77 \mathrm{~K}$ and $4 \mathrm{MPa}$, previous studies found a better correlation for the total microporosity $(<2 \mathrm{~nm})[12,23]$. Accordingly, in Fig. $6 \mathrm{~b}$ the maximum adsorption amounts at $77 \mathrm{~K}$ are plotted over the total micropore volume, $\mathrm{V}_{D R}\left(\mathrm{~N}_{2}\right)$ (for samples with $\mathrm{V}_{D R}\left(\mathrm{CO}_{2}\right)>\mathrm{V}_{D R}\left(\mathrm{~N}_{2}\right), \mathrm{V}_{D R}\left(\mathrm{CO}_{2}\right)$ was used instead). Also at $77 \mathrm{~K}$ the expected linear relationships with micropore volumes larger than $0.1 \mathrm{~cm}^{3} \mathrm{~g}^{-1}$ can be observed, being in agreement with previously reported results [12, 23-28].

In difference to other studies, in which adsorbents of various origins were compared, our results were obtained for materials which all derived from similar sources (coal tar pitch CFs). This type of similarity indicates that, upon $\mathrm{KOH}$ activation, the pristine structure of the precursor material has little influence on hydrogen uptake results, due to the considerable modification of its porosity upon activation.

In addition to the gravimetric adsorption amounts, we also correlate the excess adsorption on a volumetric basis in Fig. 6 The volumetric adsorption amounts were calculated by multiplication of the gravimetric values with the corresponding packing densities from Table 1 . In contrast to what was found for the gravimetric values at both adsorption temperatures, no linear trend can be observed for the volumetric adsorption amounts. Interestingly, these results clearly indicate that the activation extend should be restricted and that highly developed adsorbents do not need to be developed to obtain maximum values in terms of volumetric adsorption. Instead, an optimum relation between developed porosity and packing density needs to be found. 


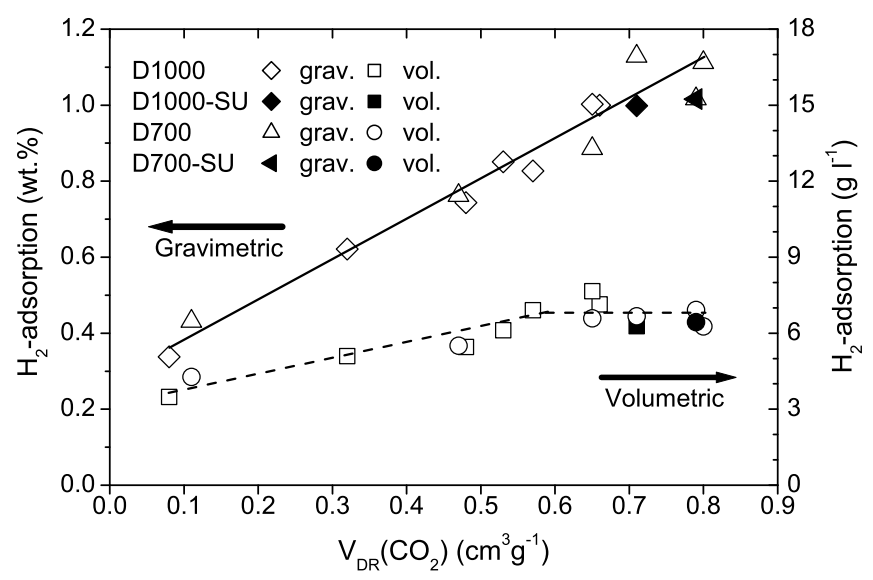

(a) $298 \mathrm{~K}$ and $20 \mathrm{MPa}$

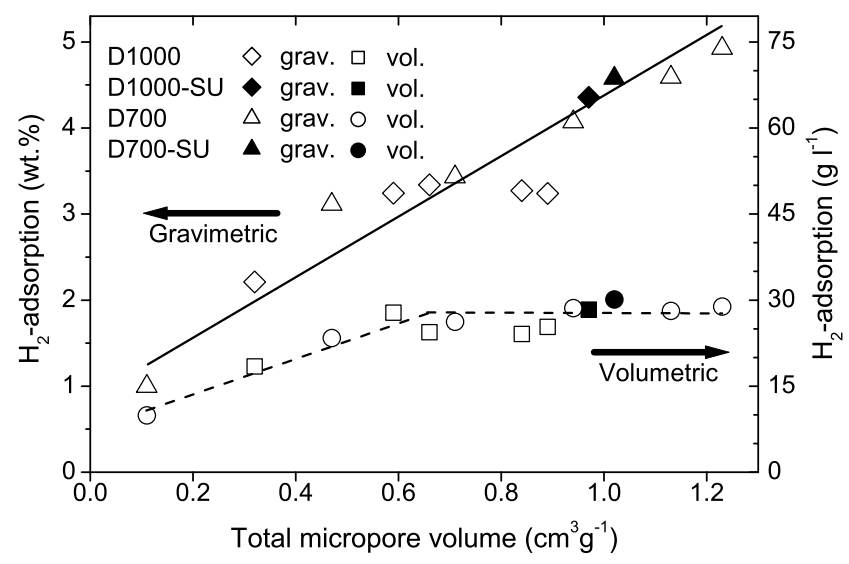

(b) $77 \mathrm{~K}$ and $4 \mathrm{MPa}$

Figure 6: Maximum excess adsorption of hydrogen over micropore volumes. Adsorption amounts are given on gravimetric basis (left ordinates) and volumetric basis (right ordinates). Values correspond to samples that are based on D700 (circles) and D1000 (squares) precursors. Empty symbols indicate lab-scale activations, filled symbols indicate scale-up activations. (a) Results obtained at room temperature and up to $20 \mathrm{MPa}$ over narrow micropore volume, $\mathrm{V}_{D R}\left(\mathrm{CO}_{2}\right)$; (b) Results obtained at $77 \mathrm{~K}$ and up to $4 \mathrm{MPa}$ over total micropore volume.

Until now, only gravimetric and volumetric excess adsorption of $\mathrm{H}_{2}$ has been investigated. However, for practical applications, it is of interest which amount of hydrogen can be stored inside a determined tank design. If we assume that the volume of the tank is filled with an adsorbent of finite density, then the hydrogen molecules can reside at two possible locations: They can (i) be adsorbed on the surface of the adsorbent, or (ii) consist as a compressed gas in the void space in-between the adsorbent particles. Theoretically, an ideal case would be if the void space would approximate zero. Then all of the stored hydrogen would consist as adsorbate, which has a higher density than the gas phase. The contrary case would be a void space of $100 \%$, implying an empty tank. Real adsorbents always contain certain amount of void space, and its contribution to the total storage needs to be taken into account in order to estimate the total storage capacity of a material. Therefore, the effort has to be made to reduce the void space as much as possible by increasing the packing density of the material. The void space can be estimated by means of the following formula: $V_{\text {Void }}=1-\left(\delta_{\text {Pack }} / \delta_{\text {Skel }}\right)\left[1,12,23,24\right.$, ,44], where $V_{\text {Void }}$ is the void space, $\delta_{\text {Pack }}$ the packing density, and $\delta_{S k e l}$ the skeleton density of the material. In Table 2, we applied the above equation to the maximum $\mathrm{H}_{2}$ adsorption amounts for each sample. Thereby, the corresponding packing densities, $\delta_{\text {Pack }}$, from Table 11 were used, and it was assumed that the skeleton density, $\delta_{\text {Skel }}$, is equal to the density of graphite $\left(2.22 \mathrm{~g} \mathrm{~cm}^{-3}\right)$ [14]. The highest values that were obtained are interestingly high: $17.1 \mathrm{~g} \mathrm{l}^{-1}$ at $298 \mathrm{~K}$, and $38.6 \mathrm{~g} \mathrm{l}^{-1}$ at $77 \mathrm{~K}$, respectively. Clearly, not only the porosity of the material, but also its packing density is of particular importance for the total amount of hydrogen stored. In general, higher storage amounts are reached at $77 \mathrm{~K}$ than at $298 \mathrm{~K}$, the D1000 precursor being the only exception. Due to the lack of hydrogen adsorption at $77 \mathrm{~K}$ for this material, the only contribution to the total storage amount comes from the compression inside the void space.
Table 2: Total hydrogen storage amounts of the investigated samples

\begin{tabular}{lcc}
\hline Sample & $\begin{array}{c}\mathrm{H}_{2 ; \text { total }} \text { at } 298 \mathrm{~K} \\
\mathrm{~g} \mathrm{l}^{-1}\end{array}$ & $\begin{array}{c}\mathrm{H}_{2 ; \text { total }} \text { at } 77 \mathrm{~K} \\
\mathrm{~g} \mathrm{l}^{-1}\end{array}$ \\
\hline D1000 & 11.1 & 7.3 \\
D1000-K1 & 14.1 & 23.7 \\
D1000-K2 & 15.0 & - \\
D1000-K3 & 15.8 & 35.7 \\
D1000-K4 & 16.8 & 32.2 \\
D1000-K6 & 16.9 & 33.3 \\
D1000-K10 & 15.8 & 32.6 \\
\hline D1000-K6-SU & 16.5 & 37.2 \\
\hline D700 & 12.2 & 15.4 \\
D700-K1 & 15.2 & 30.8 \\
D700-K2 & 16.1 & 34.1 \\
D700-K3 & 16.7 & 37.1 \\
D700-K4 & 17.1 & 37.4 \\
D700-K5 & 16.9 & 38.2 \\
\hline D700-K3-SU & 16.6 & 38.6 \\
\hline
\end{tabular}

Continuing with this concept of total hydrogen storage, and expressed on a gravimetric basis, all the data of Fig. 4 and 5 reaches considerably higher total hydrogen capacities than the plotted values. As an example, the two highest values obtained at $298 \mathrm{~K}(1.1 \mathrm{wt} . \%)$ and at $77 \mathrm{~K}(4.9 \mathrm{wt} . \%)$, corresponding to samples D700-K4 in Fig. (4)and to D700-K5 in Fig. 5, give total hydrogen capacities of $2.8 \mathrm{wt} . \%$ and $6.4 \mathrm{wt} . \%$, respectively.

Often, such storage densities on a materials basis are directly compared with the DOE milestones mentioned in the Introduction Section. By doing so, the above mentioned maximum values obtained at $77 \mathrm{~K}$ would suggest the exceeding of the 2010 DOE milestones for light-duty vehicles, and the approximation to those scheduled for 2015. However, these comparisons are not precise and can only serve as a rough orientation, be- 
cause, as mentioned earlier, the milestones refer to the whole storage system. Thus, in the case of adsorbent materials, one would have to add the weight and volume of the tank shell, rigid enough for withstanding the nominal pressure, and, in the case of cryogenic conditions, the cooling system. In addition, the use of cryogenic coolant indirectly affects other system requirements, e.g. the fuel costs or possible hydrogen loss due to long-term parking periods.

Our findings indicate that large amounts of hydrogen storage materials can be synthesized by chemical activation of CFs. However, further studying has to be performed regarding the process parameters, in order to produce optimized hydrogen storage materials. For example, the carbonization temperature of the precursor has a high impact on the process, a fact which, up to now, few allowance has been made for.

\section{Conclusions}

From the work carried out with two types of coal-tar pitch based carbon fibre materials (CF), chemically activated with $\mathrm{KOH}$, and their hydrogen adsorption isotherms, measured at $298 \mathrm{~K}$ and $77 \mathrm{~K}$, the following conclusions are obtained:

- The lower carbonization temperature of one of the precursors (D700) turned out to be significantly beneficial for the synthesis of hydrogen storage materials by activation with $\mathrm{KOH}$. Thus, samples with higher apparent BET surface areas, and higher narrow microporosity were obtained with this precursor. Thereby, the process yield was only slightly lower.

- The synthesized materials performed satisfyingly in hydrogen adsorption measurements. Thus, gravimetric excess adsorption amounts of $1.1 \mathrm{wt}$ \% were reached at room temperature and high pressures up to $20 \mathrm{MPa}$. At $77 \mathrm{~K}$ and up to $4 \mathrm{MPa}$ the maximum adsorption amount reached around $5 \mathrm{wt} . \%$.

- A scale-up activation approach was performed successfully for both CF precursors. Thereby, the amount of activated material was increased by more than one order of magnitude. Highly microporous materials were obtained by scale-up activation. An apparent BET surface area of more than $2300 \mathrm{~m}^{2} \mathrm{~g}^{-1}$ and a narrow micropore volume of up to $0.8 \mathrm{~cm}^{3} \mathrm{~g}^{-1}$ were reached by this process, having a yield as high as $60 \%$. The obtained results show that there is no problem in carrying out a scale-up in the studied range.

- The hydrogen uptakes of the materials that were synthesized by scale-up activation were comparable (in the case of the D1000 precursor even better) with the samples that were synthesized on lab-scale. Thus, hydrogen excess adsorption amounts of around $1 \mathrm{wt} . \%$ and $4.5 \mathrm{wt} . \%$ were obtained at $298 \mathrm{~K}$ and at $77 \mathrm{~K}$, respectively.

- It is shown that the packing density of the adsorbent is extremely important in order to estimate the total amount of hydrogen which, in practice, can be stored in a determined tank volume. For the synthesized ACFs, total volumetric storage amounts of $17.1 \mathrm{~g} \mathrm{l}^{-1}$ at $298 \mathrm{~K}$, and $38.6 \mathrm{~g} \mathrm{l}^{-1}$ at $77 \mathrm{~K}$ were calculated, respectively.

\section{Acknowledgements}

The authors thank Osaka Gas Co., Ltd. for supplying the two Donacarbo samples, and the European Union (Marie Curie Research Training Network - HyTRAIN - Project reference: 512443), MEC (ENE2005-23824-E/CON), the Generalitat Valenciana (ACOMP06/089 and PROMETEO/2009/047), as well as MEC-CTQ2006-08958/PPQ for financial help.

\section{References}

[1] Kunowsky M, Weinberger B, Lamari Darkrim F, Suárez-García F, Cazorla-Amorós D, Linares-Solano A. Impact of the carbonisation temperature on the activation of carbon fibres and their application for hydrogen storage. International Journal of Hydrogen Energy 2008;33(12):3091-95.

[2] Schlapbach L. Technology: Hydrogen-fuelled vehicles. Nature 2009;460:809-11.

[3] Basic Research Needs for the Hydrogen Economy (2004) Office of Basic Energy Sciences, U.S. Department of Energy, Washington, DC. http://www.sc.doe.gov/bes/hydrogen.pdf

[4] von Helmolt R, Eberle U. Fuel cell vehicles: Status 2007. Journal of Power Sources 2007;165(2):833-43

[5] Targets for Onboard Hydrogen Storage Systems for Light-Duty Vehicles (2009) Revision 4.0, September 2009, U.S. Department of Energy, Washington, DC.

http://www1.eere.energy.gov/hydrogenandfuelcells/ storage/pdfs/targets_onboard_hydro_storage_ explanation.pdf

[6] Wallner T, Lohse-Busch H, Gurski S, Duoba M, Thiel W, Martin D, Korn T. Fuel economy and emissions evaluation of BMW Hydrogen 7 MonoFuel demonstration vehicles. International Journal of Hydrogen Energy 2008;33(24):7607-18.

[7] Poirier E, Chahine R, Bénard P, Cossement D, Lafi L, Mélançon E, Bose T, Désilets S. Storage of hydrogen on single-walled carbon nanotubes and other carbon structures. Applied Physics A: Materials Science and Processing 2004;78(7):961-7.

[8] Pfeifer P, Wall C, Jensen O, Hahn H, Fichtner M. Thermal coupling of a high temperature PEM fuel cell with a complex hydride tank. International Journal of Hydrogen Energy 2009;34(8):3457-66.

[9] Veerraju Ch, Ram Gopal M. Heat and mass transfer studies on elliptical metal hydride tubes and tube banks. International Journal of Hydrogen Energy 2009;34(10):4340-50.

[10] Bérubé V, Radtke G, Dresselhaus M, Chen G. Size effects on the hydrogen storage properties of nanostructured metal hydrides: A review. International Journal of Energy Research 2007;31(6-7):637-63.

[11] Bhatia SK, Myers AL. Optimum conditions for adsorptive storage. Langmuir 2006;22(4):1688-700.

[12] Linares-Solano A, Jordá-Beneyto M, Kunowsky M, Lozano-Castelló D, Suárez-García F, Cazorla-Amorós D, 2008. Hydrogen Storage in Carbon Materials, in: Terzyk AP, Gauden PA, Kowalczyk P (Eds.), Carbon Materials - Theory and Practice. Research Signpost, Kerala, pp. 245-81.

[13] Bansal RC, Donnet JB, Stoeckli HF, 1988. Active Carbon, Marcel Dekker, New York.

[14] Pierson HO, 1993. Handbook of carbon, graphite, diamond and fullerenes: properties, processing and applications, Noyes Publications, Park Ridge, New Jersey, USA.

[15] Rodríguez-Reinoso F. The role of carbon materials in heterogeneous catalysis. Carbon 1998;36(3):159-75.

[16] Marsh H, Heinz EA, Rodríguez-Reinoso F, 1997. Introduction to Carbon Technologies, University of Alicante, Alicante. 
[17] Linares-Solano A, Lozano-Castelló D, Lillo-Ródenas MA, CazorlaAmorós D. Carbon activation by alkaline hydroxides preparation and reactions, porosity and performance. Chemistry and Physics of Carbon 2008;30:1-62.

[18] Ströbel R, Garche J, Moseley PT, Jörissen L, Wolf G. Hydrogen storage by carbon materials. Journal of Power Sources 2006;159(2):781-801.

[19] Noh JS, Agarwal RK, Schwarz JA. Hydrogen storage systems using activated carbon. International Journal of Hydrogen Energy 1987;12(10):693-700.

[20] Chahine R, Poirier E, Bose TK. Hydrogen adsorption in carbon nanostructures. International Journal of Hydrogen Energy 2001;26(8):831-5.

[21] Xu WC, Takahashi,K, Matsuo Y, Hattori Y, Kumagai M, Ishiyama $\mathrm{S}$, Kaneko K, Iijima S. Investigation of hydrogen storage capacity of various carbon materials. International Journal of Hydrogen Energy 2007;32(13):2504-12.

[22] Cabria I, López MJ, Alonso JA. Hydrogen storage capacities of nanoporous carbon calculated by density functional and Møller-Plesset methods. Physical Review B - Condensed Matter and Materials Physics 2008;78(7):075415.

[23] Jordá-Beneyto M, Suárez-García F, Lozano-Castelló D, Cazorla-Amorós D, Linares-Solano A. Hydrogen storage on chemically activated carbons and carbon nanomaterials at high pressures. Carbon 2007;45(2):293-303.

[24] De La Casa-Lillo MA, Lamari-Darkrim F, Cazorla-Amorós D, LinaresSolano A. Hydrogen storage in activated carbons and activated carbon fibers. Journal of Physical Chemistry B 2002;106(42):10930-4.

[25] Texier-Mandoki N, Dentzer J, Piquero T, Saadallah S, David P, VixGuterl C. Hydrogen storage in activated carbon materials: Role of the nanoporous texture. Carbon 2004;42(12-13):2735-47.

[26] Thomas KM. Hydrogen adsorption and storage on porous materials. Catalysis Today 2007;120(3-4):389-98.

[27] Panella B, Hirscher M, Roth S. Hydrogen storage in different carbon nanostructures. Carbon 2005;43(10):2209-14.

[28] Gogotsi Y, Portet C, Osswald S, Simmons JM, Yildirim T, Laudisio G, Fischer JE. Importance of pore size in high-pressure hydrogen storage by porous carbons. International Journal of Hydrogen Energy 2009;34(15):6314-9.

[29] Nijkamp MG, Raaymakers JEMJ, Van Dillen AJ, De Jong KP. Hydrogen storage using physisorption-materials demands. Applied Physics A: Materials Science and Processing 2001;72(5):619-23.

[30] Züttel A, Sudan P, Mauron Ph, Kiyobayashi T, Emmenegger Ch, Schlapbach L. Hydrogen storage in carbon nanostructures. International Journal of Hydrogen Energy 2002;27(2):203-12.

[31] Zubizarreta L, Arenillas A, Pis JJ. Carbon materials for $\mathrm{H}_{2}$ storage. International Journal of Hydrogen Energy 2009;34(10):4575-81.

[32] Eberle U, Felderhoff M, Schüth F. Chemical and physical solutions for hydrogen storage. Angewandte Chemie - International Edition 2009;48(36):6608-30.

[33] Van Den Berg AWC, Areán CO. Materials for hydrogen storage: Current research trends and perspectives. Chemical Communications 2008;6:66881.

[34] Zhou L. Progress and problems in hydrogen storage methods. Renewable and Sustainable Energy Reviews 2005;9(4):395-408.

[35] Zabaniotou A, Stavropoulos G, Skoulou V. Activated carbon from olive kernels in a two-stage process: Industrial improvement. Bioresource Technology 2008;99:320-6.

[36] Eigen N, Keller C, Dornheim M, Klassen T, Bormann R. Industrial production of light metal hydrides for hydrogen storage. Scripta Materialia 2007;56(10):847-51.

[37] Wigmans T. Industrial aspects of production and use of activated carbons. Carbon 1989;27(1):13-22.

[38] El Qada EN, Allen SJ, Walker GM. Influence of preparation conditions on the characteristics of activated carbons produced in laboratory and pilot scale systems. Chemical Engineering Journal 2008;142(1):1-13.

[39] Lopes FVS, Grande CA, Ribeiro AM, Oliveira ELG, Loureiro JM, Rodrigues AE. Enhancing capacity of activated carbons for hydrogen purification. Industrial and Engineering Chemistry Research 2009;48(8):397890.

[40] Cazorla-Amorós D, Alcaniz-Monge J, Linares-Solano A. Characterization of activated carbon fibers by $\mathrm{CO}_{2}$ adsorption. Langmuir 1996;12(11):2820-4.

[41] Gadea-Ramos E, Suárez-García F, Cazorla-Amorós D, Jordá-Beneyto
M, Linares-Solano A. Method and equipment for measuring the highpressure sorption isotherms of supercritical fluids and gases. International patent, WO 2008/107505 (A1), September 12, 2008.

[42] Lozano-Castelló D, Lillo-Ródenas MA, Cazorla-Amorós D, LinaresSolano A. Preparation of activated carbons from Spanish anthracite: I. Activation by KOH. Carbon 2001;39(5):741-49.

[43] Lozano-Castelló D, Calo JM, Cazorla-Amorós D, Linares-Solano A. Carbon activation with $\mathrm{KOH}$ as explored by temperature programmed techniques, and the effects of hydrogen. Carbon 2007;45(13):2529-36.

[44] Zhou L, Zhou Y, Sun Y. Enhanced storage of hydrogen at the temperature of liquid nitrogen. International Journal of Hydrogen Energy 2004;29(3):319-22. 